Nature Reviews Nephrology 10, 542 (2014); published online 19 August 2014; doi:10.1038/nrneph.2014.152; doi:10.1038/nrneph.2014.154; doi:10.1038/nrneph.2014.151; doi:10.1038/nrneph.2014.153

\section{IN BRIEF}

\section{DIALYSIS}

Haemodialysis-associated cardiovascular risks revealed

Haemodialysis improves some aspects of cardiovascular health, but whether home (5-6 times per week) or in-centre (3 times per week) haemodialysis is more effective in this regard is unknown. The risk of hospitalization and mortality due to all causes were similar in patients receiving home $(n=3,480)$ or in-centre $(n=17,400)$ haemodialysis. The home haemodialysis group had significantly lower risk of hospitalization with cardiovascular disease, but significantly greater risk with infection, which suggests better prevention of infection is required in patients on home haemodialysis.

Original article Weinhandl, E. D. et al. Hospitalization in daily home hemodialysis and matched thrice-weekly in-center hemodialysis patients. Am. J. Kidney Dis. doi:10.1053/j.ajkd.2014.06.015

\section{GENETICS}

Rare mutations associated with chronic kidney disease Through screening of 24 million different single nucleotide polymorphisms in a large Icelandic study population, previously unidentified mutations in three solute carrier genes (SLC6A19, SLC25A45 and SLC47A1) and two E3 ubiquitin ligase genes (RNF186 and RNF128) were found. Association studies with serum creatinine showed these mutations explained $0.5 \%$ of the variability in serum creatinine among Icelanders. Further analysis (15,594 cases and 291,428 controls) revealed that three of these variants were associated with chronic kidney disease.

Original article Sveinbjornsson, G. et al. Rare mutations associating with serum creatinine and chronic kidney disease. Hum. Mol. Genet. doi:10.1093/hmg/ddu399

\section{POLYCYSTIC KIDNEY DISEASE}

High-resolution ultrasonography improves cyst detection

Ultrasonography is currently used to diagnose autosomal dominant polycystic kidney disease, despite accuracy being limited in patients $<30$ years of age. A new study in 155 individuals showed that high-resolution ultrasonography achieved a diagnostic sensitivity of $97 \%$. Use of highresolution ultrasonography compared favourably with the reported $82 \%$ diagnostic sensitivity achieved with standard ultrasonography. This research suggests that highresolution ultrasonography is comparable in sensitivity to MRI (which has 100\% diagnostic sensitivity).

Original article Pei, Y. et al. Imaging-based diagnosis of autosomal dominant polycystic kidney disease. J. Am. Soc. Nephrol. doi:10.1681/ASN.2014030297

\section{DIABETIC NEPHROPATHY}

\section{NIrp3-inflammasome activated in diabetic nephropathy}

Inflammation is an important, yet poorly understood cause of renal damage in diabetic nephropathy. NIrp3-inflammasome activation has now been demonstrated in endothelial cells and podocytes from patients with diabetes in vitro, and in mouse models of diabetes. NIrp3-inflammasome-mediated damage did not arise from circulating immune cells. NIrp3 or CASP1 knock out and IL-1 receptor antagonism all protected against diabetic nephropathy in mouse models of diabetes. This research identifies several potential targets for diabetic nephropathy treatment.

Original article Shahzad, K. et al. Nlrp3-inflammasome activation in non-myeloidderived cells aggravates diabetic nephropathy. Kidney Int. doi:10.1038/ki.2014.271 